\title{
FATORES SOCIOECONÓMICOS Y MORTALIDAD INFANTIL EN ECUADOR, $1970-1981$
}

\author{
Guillermo González Pérez* \\ Ana María Gálvez González* \\ Melfa Jaramillo Villarreal**
}

GONZÁLES PÉREZ, G. et al. Fatores socioeconómicos y mortalidad infantil en Ecuador, 1970-1981.

Rev. Saúde públ., S. Paulo, 22:273-80, 1988.

RESUMEN: En Ecuador la tasa de mortalidad infantil se redujo entre 1970 y 1981 en un $35 \%$, a pesar de lo cual su valor sigue siendo elevado en el contexto latinoamericano. A nivel provincial, el comportamiento de la tasa no ha sido homogéneo, observándose fuertes diferencias entre las entidades en cuanto a la mortalidad durante el primer año de vida. Mediante el empleo de técnicas de regresión múltiple, pretendiose identificar aquellos factores socioeconómicos que más han incidido en el descenso de la mortalidad infantil en Ecuador en el periodo señalado, así como las variables que más explican las diferencias interprovinciales en dicho indicador. La disminución de la natalidad y el aumento proporcional de los gastos del presupuesto destinado a salud por una parte, y la tasa de alfabetización por otra parecen ser elementos claves para una explicación económico social de los cambios recientes en la mortalidad infantil ecuatoriana.

DESCRIPTORES: Mortalidad infantil, tendencias. Factores socioeconomicos.

\section{INTRODUCCIÓN}

El nivel de la mortalidad infantil no sólo depende de la atención médica que reciben la madre o el niño, sino también de diversos factores socioeconómicos, biodemográficos y ambientales; por tanto, valores elevados en este indicador vienen a expresar en última instancia, la poca eficiencia de la estructura económicosocial para lograr que los infantes arriben con vida a su primer cumpleaños. En América Latina, pocos son los países - Cuba, Costa Rica - que exhiben bajas tasas de mortalidad infantil ${ }^{1}$. Ecuador no es la excepción. Si bien su tasa ha experimentado un incuestionable descenso entre 1970 y 1985; aún presenta cifras superiores a 40 por cada mil habitantes (sin contar el posible subregistro existente), predominando proporcionalmente aquellas defunciones debidas a causas factibles de evitar con diversas medidas higiénico sociales.

Tras la bonanza petrolera de los años 70 , Ecuador se ha sumido en una crisis económica - agravada por situaciones políticas y climatológicas - en a cual la baja del precio del crudo en el mercado internacional y la creciente deuda externa desempeñam un papel fundamental. Estos aspectos, lógicamente, repercuten en el desarrollo social del país y especialmente, en el nivel de salud de la población, en particular, la infantil.

Por otra parte, el desarrollo alcanzado por las distintas regiones del país es indudablemente desigual, concentrándose recursos y servicios en algunas provincias de la Sierra y la Costa, sobre todo en aquellas donde se asientan las principales ciudades del país, en detrimento del resto de las áreas, lo que por supuesto, se refleja en los principales indicadores de salud entre ellos, la mortalidad en el primer año de vida.

Ante tal situación, resulta necesario indagar con mayor profundidad en las condicionantes socioeconómicas de la mortalidad infantil en el Ecuador. El presente trabajo va encaminado a determinar en primer lugar, cuáles han sido las variables socioeconómicas y demográficas que han ejercido una mayor influencia en la evolución de la mortalidad infantil en Ecuador entre 1970 y 1981 y además identificar aquellas variables que más han incidido en las diferencias interprovinciales observadas en la mortalidad infantil para los años 1974 y 1982.

\footnotetext{
* Facultad de Salud Publica. Instituto Superior de Ciencias Médicas de La Habana. Calle 146, n: 2504 entre 25 y 31 . Cubanacán. Municipio Playa. Apartadó de Correos n? 14063. Zona Postal n? 14 - La Habana. Cuba.

** Dirección Provincial de Salud en Chimborazo, Ecuador.
} 


\section{MATERIAL Y METODO}

Para analizar la relación existente entre las condiciones socioeconómicas y la evolución de la mortalidad infantil se seleccionó un conjunto de variables para el período 1970-1981, único en el cual estaba disponible toda la información socioeconómica del país.

Las variables seleccionadas fueron:

X1 Tasa de alfabetización (población de 15 años y más).

X2 Grado de urbanización.

X3 Consumo de electricidad percápita.

X4 Exportación de petróleo crudo y sus derivados (dólares/habitante).

X5 Exportación de productos (excluyendo petróleo y sus derivados) (dólares/habitante).

X6 Producto Interno Bruto percápita.

X7 Reforma Agraria (hectáres entregadas por familia).

X8 Porciento de población cotizante en el Instituto Ecuatoriano de Seguridad Social.

X9 Importación de productos farmacéuticos (dólares/habitante).

X10 Tasa bruta de natalidad (nacidos vivos por mil habitantes).

$\mathrm{X} 11$ Porciento del presupuesto nacional dedicado a salud.

X12 Tasa de inflación.

No se contaba con información para todo el período de las dos últimas variables, por lo cual se incluyeron en una segunda fase donde se analizó el período 1972-1980.

Con esta amplia gama de variables se pretende tener una idea de las condiciones que han podido influir en el comportamiento de la mortalidad infantil; la mayoría de ellas responde a asociaciones con frecuencia vistas en la literatura sobre el tema y otras a la situación propia del país. La introducción de una variable referida a la Reforma Agraria, por ejemplo, está en relación con la importancia del desarrollo agrario en el país, donde más de la mitad de la población vive en zonas rurales.

Para el análisis a nivel provincial se utilizó un conjunto de variables socioeconómicas disponibles de cada provincia para los años 1974 y 1982 (años censales) que se considera pudieran influir sobre el comportamiento de la tasa de mortalidad infantil además de ser, en cierta medida, indicadores del desarrollo alcanzado por cada una de estas entidades.

Estas son:

X1 Tasa de alfabetización.
X2 Grado de urbanización.

X3 Tasa de natalidad.

$\mathrm{X} 4$ Abonados por servicios telefónicos (por mil habitantes).

X5 Habitantes por emisoras radiales.

Para el análisis estadístico de la información se utilizó un modelo de regresión lineal múltiple.

El método escogido para seleccionar la mejor ecuación de regresión, es decir, la que incluyera las variables de mayor peso o importancia es el conocido como Paso a Paso (Stepwise) ${ }^{2}$, uno de los más completos para este tipo de análisis.

Este método analiza en cada etapa las variables que ya se habian incorporado al modelo en fases anteriores, porque una variable que pudo ser la mejor para entrar en una etapa, puede, en una posterior ser innecesaria debido a las relaciones entre ella y el resto de las variables que están en la ecuación.

A nivel provincial se realizó un análisis de residuos, el cual brinda la posibilidad de discutir, con los datos de 1974 y 1982, las posiciones relativas de las provincias analizadas luego de controlar el efecto de las variables que intervendrán en el estudio.

La información utilizada se obtuvo del manual de indicadores socioecoriómicos del Consejo Nacional de Desarrollo ${ }^{3}$ (CONADE) (datos socioeconómicos) y de los Anuarios de Estadísticas Vitales ${ }^{4}$ y de los Censos de Población y Vivienda del Ecuador ${ }^{5}$, editados por el Instituto de Estadísticas y Censos del Ecuador (INEC) (datos demográficos).

\section{ANALISIS Y DISCUSION}

\section{Las Variables Socioeconómicas y la Evolución de la Mortalidad Infantil en el Ecuador}

Es un hecho cierto que la mortalidad infantil en el Ecuador ha descendido entre 1970 y 1981. Las estadísticas oficiales, si bien subestiman el nivel real de la mortalidad en la población infantil ${ }^{6}$, permiten apreciar una indudable reducción de la tasa, que de $76,6 \%$ a inicios de la década, se sitúa en $50,5 \%$ en 1981 , una disminución del $35 \%$ con respecto a 1970 .

El desarrollo social es un elemento determinante para lograr un descenso de la mortalidad en el primer año de vida. En tal sentido, la búsqueda de argumentos que contribuyan a explicar, en el contexto ecuatoriano, este comportamiento de la tasa requiere relacionar una serie de indicadores socioeconómicos y demográficos (previamente mencionados) con la mortali- 
dad infantil. Los resultados de este análisis se presentan a continuación.

En la Tabla 1 se puede apreciar la fuerte asociación que existe entre la tasa de natalidad y la mortalidad infantil, pues sólo esta variable cumple con los requisitos establecidos para entrar en la ecuación que explica el comportamiento de la mortalidad infantil.

TABLA 1

Mortalidad infantil y variables socioeconómicas seleccionadas. Analisis đe regresion múltiple. Método paso a paso. Ecuador, 1970-1981

Paso no 1

Variables que entraron en la ecuación

\begin{tabular}{lcccc}
\hline Nombre & $\begin{array}{c}\text { Coeficiente de } \\
\text { regresión }\end{array}$ & $\begin{array}{c}\text { Coeficiente de } \\
\text { determinación }\end{array}$ & $\begin{array}{c}\text { Error } \\
\text { standard }\end{array}$ & $\begin{array}{c}\text { F } \\
(1.10)\end{array}$ \\
\hline Tasa bru ta de natalidad & 4.12 & 0.90 & 0.43 & 92.404
\end{tabular}

Variables que no entraron en la ecuación

\begin{tabular}{lccc}
\hline Nombre & $\begin{array}{c}\text { Correlación } \\
\text { parcial }\end{array}$ & F para entrar & Probabilidad \\
\hline Tasa de alfabetización & 0.0742 & 0.721 & 0.4179 \\
Urbanización & 0.1203 & 1.231 & 0.2959 \\
Consumo de electricidad & 0.1258 & 1.295 & 0.2845 \\
Exportación de petróleo & 0.1379 & 1.440 & 0.2608 \\
Exportación & 0.2445 & 2.913 & 0.1220 \\
P.I.B. & 0.1093 & 1.105 & 0.3207 \\
Reforma Agraria & 0.1176 & 1.199 & 0.3019 \\
Cotizantes IESS & 0.1126 & 1.142 & 0.3130 \\
Importación prod. farmacéuticos & 0.1205 & 1.233 & 0.2956 \\
\hline
\end{tabular}

En 1970 la tasa de natalidad fue de 38,0\% y en 1981, su valor fue de $31,6 \%$, es decir que se redujo en casi en $20 \%$, esto parece ser el resultado en gran medida de que en la década del ' 70 se promovió por diversos medios el programa de planificación familiar, cuyo financiamiento provino en lo fundamental, de organismos internacionales.

Un descenso de la natalidad y por consiguiente un menor número de nacimientos permite en cierta forma una mejor atención médica de los mismos, lo que indudablemente favorecería la sobrevivencia de una proporción mayor de infantes.

Por otra parte, la familia tiende a proteger mejor a sus hijos si estos son menos numerosos y además debe tomarse en cuenta que la reducción de la natalidad puede reflejarse en una menor proporción de nacimientos de orden elevado, muy seguidos o provenientes de madres con más de 34 años, donde el riesgo de muerte para el niño es mayor. Estos pudieran ser algunos elementos que permitirían explicar esta asociación ${ }^{7}$, aunque no debe olvidarse la posible presencia de un efecto inverso, pues la mor- talidad infantil en la medida en que disminuye puede traer aparejado un descenso de la natalidad al no estar la familia tan necesitada de tener un número elevado de hijos para reponer la posible pérdida de algunos de ellos.

Una segunda fase de este análisis incluyó a las variables tasa de inflación y porciento de presupuesto dedicado a salud, junto a otras variables analizadas previamente, pero sólo tomando en cuenta el período 1972-1980, en el cual existía información para todas estas variables.

En la Tabla 2 se puede constatar que junto a la natalidad, el incremento proporcional de los gastos del presupuesto dedicados a la salud ha desempeñado un papel relevante en el descenso de la mortalidad infantil. De hecho, ambas variables entran en la ecuación, y el coeficiente de determinación $(0,9662)$ indica que las mismas explican en gran parte el comportamiento de la tasa de mortalidad en dicho período.

De aquí se desprende la trascendencia que alcanza la proporción del presupuesto que se destina a la Salud Pública en la supervivencia de la población menor de un año. Indiscutiblemente, 
el porcentaje de presupuesto que se dedicó a salud en 1980 triplica el destinado en 1972, esto se traduce en un mayor apoyo económico para el programa materno infantil (que también ha recibido financiamiento internacional). En este programa, considerado prioritario por el Ministerio de Salud Pública, se utilizan recursos humanos y materiales del Ministerio y de la comunidad tratando de lograr una mayor cobertura; para ello se vale de fuertes estrategias que se vienen incorporando a partir de 1975 entre las cuales se pueden mencionar la capacitación del personal formal y no formal (comadronas empíricas, promotores de salud), la promoción de salud mediante publicidad, la vacunación casa a casa (1977-1983) y por último la implementación del plan de reducción de la morbimortalidad infantil (PREMI) que lleva implícito cuatro programas con el objetivo de brindar una atención integral al niño.

En definitiva debe señalarse que han sido factores en cierta medida relacionados con la atención médica - ya sea directamente (presupuesto de salud) o indirectamente (tasa de natalidad) - los que más parecen explicar la evolución de la mortalidad infantil en la década del '70 y principios de los años ' 80 .

\section{Las Variables Socioeconómicas y las Diferencias Interprovinciales en la Mortalidad Infantil}

Ahora bien, el comportamiento de la mortalidad infantil no puede considerarse homogéneo en todo el país. Existen claras diferencias interprovinciales (mantenida durante los años estudiados) en cuanto a la tasa, observándose las más altas en las provincias de la Sierra, en especial Cotopaxi, Chimborazo e Imbabura, mientras que los valores más bajos se registran en la región de la Costa - Manabí, El Oro en Pichincha (donde se ubica Quito, la capital) y en algunas zonas del Oriente. Salvo esta última región - donde la alta proporción de omisiones de fallecidos pudiera ser el motivo fundamental de tal resultado - las diferencias interprovinciales observadas parecen reflejar la desigualdad socioeconómica que caracteriza a las entidades ecuatorianas.

Es menester, por tanto, indagar sobre las variables socioeconómicas que más están diferenciando el comportamiento de la mortalidad infantil a nivel provincial en dos momentos, 1974 y 1982 . El resultado de la aplicación del método paso a paso mostró que la tasa de alfabetización (X1) es la variable de mayor peso tanto en 1974 como en 1982.

La ecuación resultante es la siguiente:

$$
\mathrm{Y}=\mathrm{b} 0+\mathrm{b} 1 \mathrm{X} 1
$$

donde

$\begin{array}{lcccc}\text { año } & \text { b0 } & & \text { b1 } & r^{2} \\ 1974 & 148.87 & - & 1.01 & 0.21 \\ 1982 & 152.44 & - & 1.21 & 0.37\end{array}$

Diversos estudios han puesto de manifiesto la intima relaciôn entre educación y mortalidad infantil, pues en la medida que aumenta el nivel de instrucción familiar, y en especial el materno, disminuye el riesgo de muerte del niño ${ }^{8}$.

En tal sentido, la tasa de alfabetización está reflejando a nivel provincial, la existencia o no de condiciones favorables para la supervivencia del infante. Es lógico inferir que a mayor alfabetización, menos mortalidad infantil, por lo que su identificación como elemento diferenciador en cuanto a la mortalidad infantil por provincias adquiere particular relevancia desde el punto de vista social, más si se tiene en cuenta el hecho de que la tasa de alfabetización fue la variable menos asociada al descenso de la mortalidad infantil en Ecuador entre 1970 y 1982.

Por otra parte, se puede apreciar que en 1982 la alfabetización explica en mayor medida las diferencias existentes en cuanto a la mortalidad infantil entre las provincias que en 1974, lo que da una idea de que el papel de esta variable lejos de disminuir se hace más notable con el tiempo.

Durante el período analizado se llevó a cabo en el país un programa de alfabetización cuyo mayor énfasis fue dirigido prioritariamente a las áreas rurales, no obstante, subsisten grandes diferencias entre las provincias en lo referente a este indicador.

Un aspecto importante a señalar radica en el hecho de que si bien en todas las provincias las tasas de mortalidad infantil disminuyeron entre 1974 y 1982 , no todas presentan las tasas que se pudieran esperar dado su desarrollo socioeconómico, lo que se evidencia en cierta medida al comparar su tasa de mortalidad infantil real con la que cabría esperar que tuviera de acuerdo a sus condiciones socioeconómicas. Este análisis se realizó evaluando el comportamiento de los residuos a partir de la experiencia de otros trabajos realizados ${ }^{9,10}$.

Como se aprecia en el mapa (Fig.) en general las provincias que presentan tasas reales inferiores a las esperadas en 1974 se comportan de forma similar en 1982, lo que también ocurre con aquellas que poseen tasas reales superiores a las esperadas en estos dos años. La excepción está dada por Pastaza, que en 1974 poseía una tasa real inferior a la esperada, lo que varió para 1982, pues en esto año su tasa real es ligeramente superior a la esperada. 
TABLA 2

Mortalidad infantil y variables socioeconómicas seleccionadas. Analisis de regresión múltiple. Método paso a paso. Ecuador, 1972-1980

Paso n? 1

Variables que entraron en la ecuación

\begin{tabular}{|c|c|c|c|c|}
\hline Nombre & $\begin{array}{l}\text { Coeficiente de } \\
\text { regresión }\end{array}$ & $\begin{array}{l}\text { Coeficiente de } \\
\text { correlación parcial }\end{array}$ & $\begin{array}{l}\text { EIror } \\
\text { standard }\end{array}$ & $\underset{(1.10)}{F}$ \\
\hline Tasa bruta de natalidad & 2.5950 & 0.7796 & 0.5632 & 21.228 \\
\hline$\%$ de presupuesto destinado a la Salud & -2.8708 & 0.7776 & 0.6269 & 20.974 \\
\hline \multicolumn{5}{|l|}{ Coeficiente de determinación $=0.9662$} \\
\hline \multicolumn{5}{|l|}{ Variables que no entraron en la ecuación } \\
\hline Nombre & $\begin{array}{c}\text { Correlación } \\
\text { parcial }\end{array}$ & F para entrar & Probabilidad & \\
\hline Urbanización & 0.0644 & 0.344 & 0.5828 & \\
\hline Tasa de alfabetización & 0.0123 & 0.062 & 0.8127 & \\
\hline Exportación & 0.1044 & 0.583 & 0.4796 & \\
\hline
\end{tabular}

TABLA 3

Provincias de Ecuador segun las tasas de mortalidad infantil ( $\left.Y_{i}\right)$ y segun los residuos ( $\left.Y_{i}-Y_{i}\right), 1974$ y 1982

\begin{tabular}{|c|c|c|c|c|c|c|c|c|}
\hline $\begin{array}{l}\text { Años } \\
\text { Orden }\end{array}$ & Provincias & $\mathbf{Y}_{\mathbf{i}}$ & $\begin{array}{c}1974 \\
\text { Provincias }\end{array}$ & $\left(\mathbf{Y}_{i}-\overline{\mathbf{Y}}_{\mathbf{i}}\right)$ & Provincias & $Y_{i}$ & $\begin{array}{c}1982 \\
\text { Provincias }\end{array}$ & $\left(\mathbf{Y}_{\mathrm{i}}-\hat{\mathrm{Y}}_{\mathrm{i}}\right)$ \\
\hline $\begin{array}{r}1 \\
2 \\
3 \\
4 \\
5 \\
6 \\
7 \\
8 \\
9 \\
10 \\
11 \\
12 \\
13 \\
14 \\
15 \\
16 \\
17 \\
18\end{array}$ & $\begin{array}{l}\text { Manabí } \\
\text { Loja } \\
\text { E1 Oro } \\
\text { Los Ríos } \\
\text { Pastaza } \\
\text { Morona S. } \\
\text { Car̃ar } \\
\text { Guayas } \\
\text { Bolívar } \\
\text { Zamora Ch. } \\
\text { Pichincha } \\
\text { Esmeraldas } \\
\text { Azuay } \\
\text { Tungurahua } \\
\text { Carchi } \\
\text { Imbabura } \\
\text { Chimborazo } \\
\text { Cotopaxi }\end{array}$ & $\begin{array}{r}38.4 \\
45.3 \\
45.5 \\
54.3 \\
59.6 \\
60.7 \\
64.4 \\
71.5 \\
71.6 \\
72.4 \\
74.9 \\
76.4 \\
84.5 \\
98.3 \\
98.7 \\
100.3 \\
105.6 \\
122.0\end{array}$ & $\begin{array}{l}\text { Manabí } \\
\text { Los Ríos } \\
\text { Loja } \\
\text { Pastaza } \\
\text { Cañar } \\
\text { El Oro } \\
\text { Morona S. } \\
\text { Bolívar } \\
\text { Esmeraldas } \\
\text { Zamora Ch. } \\
\text { Guayas } \\
\text { Azuay } \\
\text { Pichincha } \\
\text { Chimborazo } \\
\text { Imbabura } \\
\text { Tungurahua } \\
\text { Cotopaxi } \\
\text { Carchi }\end{array}$ & $\begin{array}{r}-41.3 \\
-24.3 \\
-22.4 \\
-18.2 \\
-16.1 \\
-14.4 \\
-12.1 \\
-11.2 \\
-\quad 8.0 \\
6.2 \\
8.2 \\
11.9 \\
12.3 \\
13.6 \\
16.4 \\
24.4 \\
33.5 \\
34.4\end{array}$ & $\begin{array}{l}\text { Manabí } \\
\text { El Oro } \\
\text { Morona S. } \\
\text { Loja } \\
\text { Esmeraldas } \\
\text { Pichincha } \\
\text { Bolívar } \\
\text { Azuay } \\
\text { Canaar } \\
\text { Carchi } \\
\text { Guayas } \\
\text { Los Ríos } \\
\text { Pastaza } \\
\text { Zamora Ch. } \\
\text { Imbabura } \\
\text { Tungurahua } \\
\text { Chimborazo } \\
\text { Cotopaxi }\end{array}$ & $\begin{array}{l}27.9 \\
33.7 \\
39.0 \\
39.6 \\
46.5 \\
47.2 \\
49.2 \\
50.0 \\
50.7 \\
52.6 \\
52.7 \\
52.7 \\
53.1 \\
54.2 \\
62.7 \\
64.0 \\
76.1 \\
88.0\end{array}$ & $\begin{array}{l}\text { Manabí } \\
\text { Bolívar } \\
\text { Morona S. } \\
\text { Cat̃ar } \\
\text { Esmeraldas } \\
\text { Loja } \\
\text { El Oro } \\
\text { Los Ríos } \\
\text { Pastaza } \\
\text { Azuay } \\
\text { Imbabura } \\
\text { Pichincha } \\
\text { Chimborazo } \\
\text { Carchi } \\
\text { Zamora Ch. } \\
\text { Guayas } \\
\text { Tungurahua } \\
\text { Cotopaxi }\end{array}$ & $\begin{aligned} &- 28.1 \\
&- 10.8 \\
&- 9.8 \\
&- 8.7 \\
&- 8.1 \\
&- 5.7 \\
&- 5.6 \\
&- 1.0 \\
& 0.01 \\
& 0.20 \\
& 2.2 \\
& 6.4 \\
& 7.5 \\
& 8.2 \\
& 9.3 \\
& 10.8 \\
& 12.5 \\
& 21.1\end{aligned}$ \\
\hline
\end{tabular}

Nota: No constan Napo y Galápagos. 

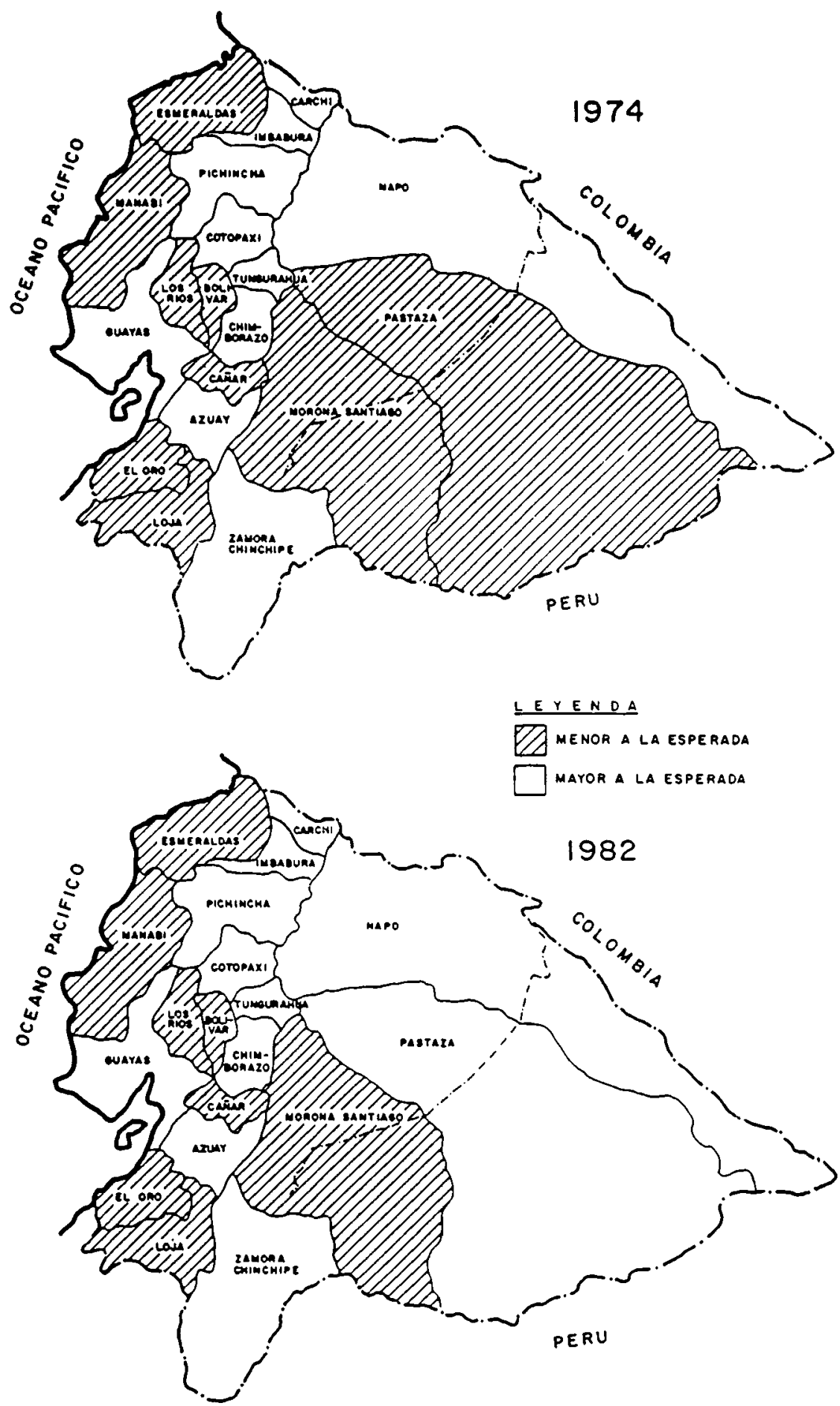

Fig. - Tasa de mortalidad infantil $1 \%$ - N.V. esperada segun sus condiciones socioeconômicas - Ecuador 1974 y 1982. 
Es muy sugerente el análisis de las provincias según su tasa de mortalidad infantil, fuera más baja o más alta que lo que cabría esperar de acuerdo a sus condiciones socioeconómicas. Cabe destacar que para los años analizados entre los cuales transcurrieron 8 años - prácticamente son las mismas provincias las que tienen tasas de mortalidad infantil mejores que las que deberían tener. Esto evidencia que en la práctica, la diferencia en cuanto a la mortalidad - y a las condicionantes sociales de las que esta depende - se mantiene en el tiempo, a pesar de las campañas de alfabetización realizadas o de otros avances sociales.

Las provincias de mayor desarrollo y por ende, con mayor alfabetización, - Guayas, Pichincha y Azuay - presentan tasas más alta que las que deberían tener, mientras otras de menor desarrollo como Morona Santiago, por ejemplo, las poseen mejores que las que les corresponderian de acuerdo con su desarrollo social. Según la literatura consultada ${ }^{3}{ }^{10}$ parece ser la gestión de salud la que explicaría en mayor medida este hecho, de lo que se desprende que en las provincias más desarrolladas los recursos humanos y materiales (más abundantes que en el resto de las provincias), no son aprovechados de forma óptima. Su relativa baja tasa en comparación con otras provincias del país descansa en sus ventajas socioeconómicas más que en un trabajo exitoso del sector Salud. (Tabla 3)

\section{CONSIDERACIONES FINALES}

- El descenso de la tasa de natalidad en el país y el incremento proporcional de los gastos del presupuesto nacional destinado a la salud, son las variables socioeconómicas que han ejercido una mayor influencia en el descenso de la tasa de mortalidad infantil observada en Ecuador entre 1970 y 1981.

- La tasa de alfabetización es la variable que más incide en las diferencias interprovinciales observadas en la mortalidad infantil, tanto en 1974 como en 1982. Para este último año el poder explicativo de esta variable es aún mayor.

- Las provincias que en 1974 presentan tasas de mortalidad infantil menores que las que cabria esperar en ellas de acuerdo con su desarrollo socioeconómico, mantienen por lo general esa situación en 1982, lo que pudiera indicar una mayor eficiencia en la gestión de salud en esas entidades.

- En las provincias de mayor desarrollo - entre ellas Guayas, Pichincha y Azuay - la tasa real es más alta que la que se podria esperar de acuerdo con sus condiciones socioeconómicas, lo que sugiere pensar que sus tasas relativamente bajas descansan más en sus ventajas socioeconómicas que en la eficiencia del sistema de salud.

- La permanencia en el tiempo de las diferencias interprovinciales en cuanto a la mortalidad infantil hace pensar en la necessidad de una política de desarrollo económico social encaminada a elevar equitativamente el nivel de vida de toda la población en las distintas regiones del país.

GONZÁLEZ PÉREZ, G. et al. Fatores sócioeconômicos e mortalidade infantil no Equador, 1970-1981.

Rev. Saúde públ., S. Paulo, 22:273-80, 1988.

RESUMO: No Equador entre os anos de 1970 a 1981 a taxa de mortalidade infantil reduziu em $35 \%$, embora seu valor continua sendo elevado em relação ao contexto latinoamericano. A nível regional, 0 comportamento da taxa não tem sido homogêneo, observando-se diferenças significativas nas diversas regiōes quanto a taxa de mortalidade durante o primeiro ano de vida. Com o emprego da técnica de regressão múltipla, pretendeu-se identificar aqueles fatores socioeconômicos que mais têm incidido no declínio da mortalidade infantil no Equador, no período acima descrito, bem como as variáveis que melhor explicam as diferenças inter-regionais do referido indicador. A diminuição da natalidade e o aumento proporcional de recursos financeiros destinados à saúde, por um lado, e a taxa de alfabetização por outro, parecem ser elementos chaves para uma explicação econômica social das mudanças recentes na mortalidade infantil equatoriana.

UNITERMOS: Mortalidade infantil, tendências. Fatores sócio-econômicos. 
GONZÁLEZ PÉREZ, G. et al. [Socioeconomic factors and infant mortality in Ecuador, 1970-1981]. Rev. Saúde públ., S. Paulo, 22:273-80, 1988.

ABSTRACT: In Equador infant mortality has dropped by $35 \%$ between 1970 and 1981, though this rate is still high in Latin American countries in general. In the interior, the behavior of the mortality rate has varied and there are some strong conırasts between them. This paper seeks to identify by multiple regression techniques, those socioeconomic factors which have determined the decline of infant mortality in the country and those variables which best explain the inter-regional differences in this indicator. The reduction of the crude birth rate and the proportional increase of health expenditure on one hand and the literacy rate on the other would seem to be the key socio-economic elements in any explanation of recent changes in Equadorian infant mortality.

UNITERMS: Infant mortality, trends. Socioeconomic factors.

\section{REFERÊNCIAS BIBLIOGRÁFICAS}

1. BEHM, H. Determinantes de la mortalidad infantil en América Latina. Rev. cub. Adm. Salud, 6, 1980. [Separata].

2. BEHM, H. \& ROSERO BIXBY, L. La mortalidad en los primeros años de vida en America Latina; Ecua. dor 1969-1970. San José, Centro Latinoamericano de Demografia, 1977. (CELADE-Série A, n: 1031)

3. BRADSHAW, J. et al. Area variations in infant mortality 1975-1977. J. Epidem. Community Hlth, 36: $11-6,1982$.

4. CONSELHO NACIONAL DE DESARROLLO (CONADE). Indicadores socioeconómicos, 1970-1981. Quito, 1982.

5. DRAPER, N.R. \& SMITH, H. Applied regression analysis. La Habana, Ed. Pueblo y Educación, 1980.

6. INSTITUTO DE ESTADÍSTICA Y CENSOS DEL ECUADOR (INEC). Anuários de estadísticas vitales, 1975, 1980 y 1985. Quito, 1975,.1980, 1985.

7. INSTITUTO DE ESTADISTICAS Y CENSOS DEL
ECUADOR (INEC). Censos de población y vivendas, 1974 y 1982 . Quito, 1974, 1982.

8. ORGANIZACIÓN PANAMERICANA DE LA SALUD. Las condiciones de salud en la Américas: 1981-1984. Washington, DC, 1986. 2 v. (OPAS Publicacion cientifica, 500).

9. ROSERO BIXBY, L. Determinantes del descenso de la mortalidad infantil en Costa Rica. Bol. Ofic. sanit. panamer., 99:510-27, 1985.

10. SILVA, L.C. et al. Evaluación de la mortalidad infantil según condiciones higiénico sociales en el municipio: un enfoque multivariado. Rev. cub. $\mathrm{Adm}$. Salud, 11(3):243-54, 1985.

Recebido para publicação em 17/11/1987 Aprovado para publicação em 29/3/1988 